\title{
Clinical efficacy and safety
}

\section{Are different hemodynamic patterns of antihypertensive drugs clinically important?}

\author{
S. Julius \\ Division of Hypertension, Department of Internal Medicine, University of Michigan Medical Center, Ann Arbor, Michigan, USA
}

\begin{abstract}
Summary. Since vascular resistance is elevated in hypertension, it is suggested that vasodilators lower the blood pressure by a physiologic mechanism and therefore must be more useful than cardiac output-lowering drugs. This is not entirely correct. Drugs that lower cardiac output are also relative vasodilators, but the vasodilation occurs at a lower level of cardiac output. It is also not necessarily true that all vasodilators are good antihypertensive agents. The clinical profile of a vasodilator depends on its effect on the venous return, cardiac output, regional blood flow, renin-angiotensin system, and sympathetic reflexes. From the viewpoint of hemodynamics, an ideal antihypertensive drug is a vasodilator that does not excessively increase cardiac output, causes no fluid retention, does not induce a great deal of venodilation, and does not elicit substantial neurohumoral counterregulation. Angiotensin-converting-enzyme inhibitors, some calcium antagonists, and some combined alpha/beta-blocking agents come close to satisfying the hemodynamic definition of an ideal antihypertensive drug.
\end{abstract}

Key words: cardiac output, vascular resistance, blood pressure

A general scheme of hemodynamics is given in Fig.1 [1]. Blood pressure is a product of cardiac output and vascular resistance, shown on the vertical and horizontal lines. The vascular resistance, shown with diagonal isoresistance lines, can be calculated by dividing the mean blood pressure by the cardiac output. It follows from the graph that the blood pressure can be decreased either by a fall in cardiac output or by a decrease in the vascular resistance. In established hypertension, high pressure is due to an increase in vascular resistance [2]. Since vasodilators diminish the high resistance, it is hypothesized that they reduce the pressure through a physiologic mechanism. Within the context of such thinking, drugs that decrease blood pres- sure through a fall in cardiac output have a physiologically undesirable effect.

Such reasoning, logical as it may be, is a great oversimplification; much depends on the circumstances. The notion that decreased cardiac output and increased vascular resistance is unhealthy will be a surprise to anybody vaguely familiar with the hemodynamic adjustment to upright posture. Cardiac output is much lower than in recumbency and the resistance is high, but reports on specific cardiovascular morbidity of upright posture are nonexistent. Similarly, a decrease in vascular resistance is not always good; hypertensive encephalopathy is associated with a great increase in cerebral blood flow [3]. Before endorsing vasodilators or condemning drugs that reduce the cardiac output, one must know much more about each of these compounds and about the prevailing conditions under which they are given.

\section{Vasodilation: at which level of cardiac output?}

The distinction between vasodilating and cardiac output lowering drugs becomes even more moot when one takes into account that the simple formula (blood pressure $=$ cardiac output $\times$ vascular resistance), while describing the physical components of the hemodynamic relationship, is not directly applicable to the living organism. Under a wide range of circumstances (upright posture, bleeding, acute intravenous beta-blockade), the fall in cardiac output is met with an increase in vascular resistance while the blood pressure remains unchanged. A pharmacologic decrease in cardiac output leads to lower pressures only if the compensatory increase in resistance fails to materialize. An example is given in Fig. 2, adapted from a study by Hansson et al. [4]. Treatment with propranolol did not decrease everybody's blood pressure but, regardless of their blood pressure responses, all patients showed a decrease in car- 


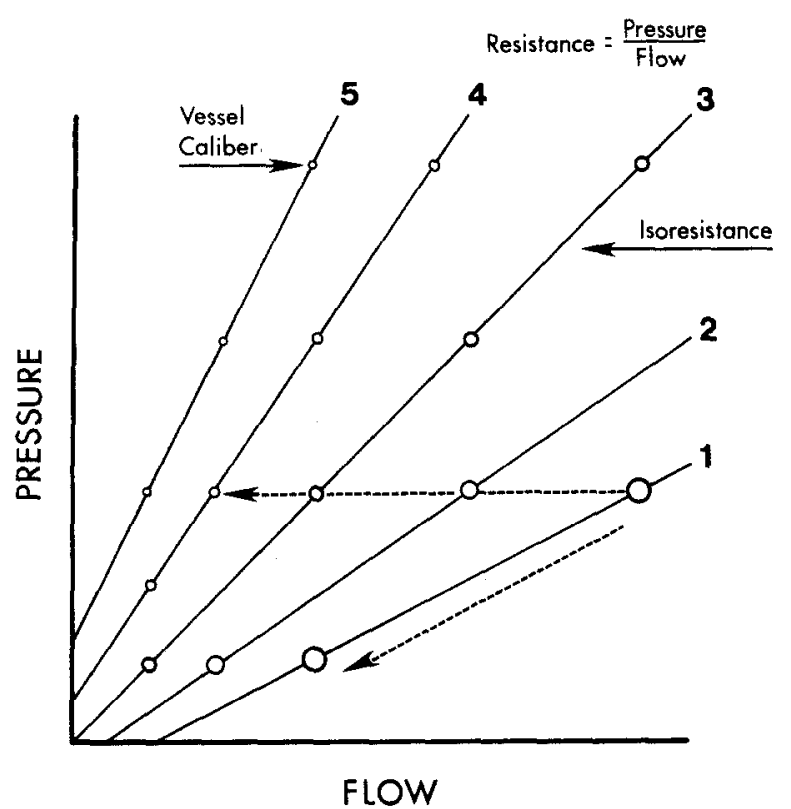

Fig. 1. The pressure-flow-resistance relationship. The diagonal lines are lines of isoresistance, with the lowest representing low resistance and the highest, high resistance. Note that the vascular caliber at low resistance is larger and that small increases in pressure cause a large increase in flow. At the high resistance line, the vascular caliber is narrow and a large increase in pressure causes only a small increase in flow. The horizontal vector represents a decrease in flow, associated with an increase in resistance and no change in pressure. The diagonal, downwardly pointing vector represents a decrease in flow, with an unchanged resistance and a fall in blood pressure

diac output. When responders and nonresponders are compared, the real difference is that responders had much lower levels of vascular resistance, which means that they were relatively more vasodilated than the nonresponders. It follows that all antihypertensive agents are vasodilators, but the dilation occurs at different levels of cardiac output.

The low cardiac output-low vascular resistance state seen with beta-adrenergic blockade is not intrinsically deleterious, but some of its clinical consequences can be disturbing. At rest and at moderate levels of exercise, the tissues continue to receive adequate oxygen supplies through increased oxygen extraction from the blood. During more strenuous exercise, beta-blocked patients cannot increase their cardiac output and experience the onset of fatigue. Good clinicians take into account this hemodynamic limitation and beta-blocking agents are

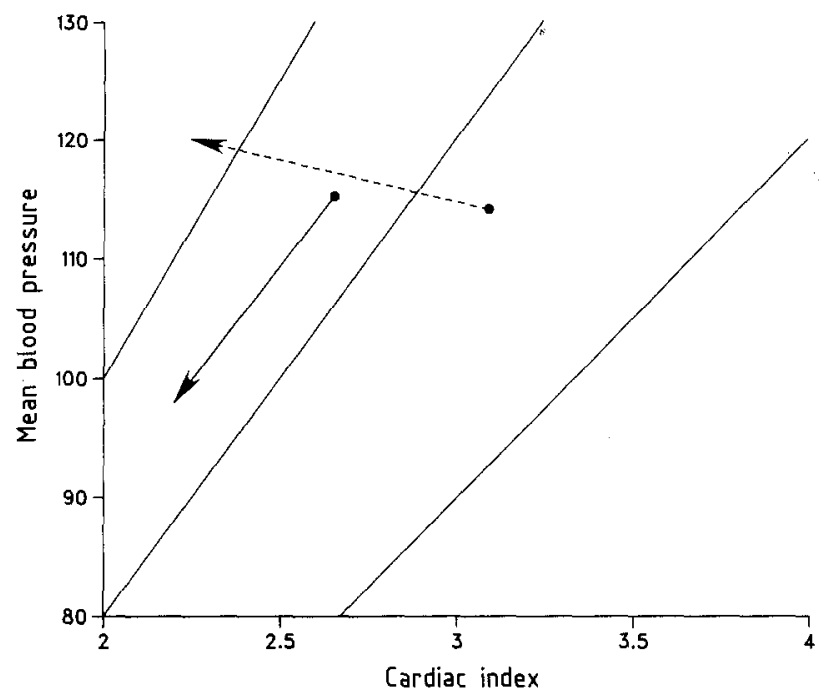

Fig.2. Hemodynamic effect of chronic beta-adrenergic blockade with propranolol. (Data adapted from Hansson et al. [4]). The patients are divided into responders (solid line) and nonresponders (broken line). The full circle at the base of the arrow presents the hemodynamic state before the treatment and the head of the arrow, the hemodynamics after 4 weeks of treatment with $160-320 \mathrm{mg}$ propranolol. Note that the non-responder group actually showed a larger decrease in the cardiac index, but this was met by an increase in vascular resistance and the blood pressure did not change. In responders, the fall of the cardiac index did not elicit an increase of resistance and the blood pressure fell. Note that on propranolol both groups had low cardiac indexes, but the responder group had substantially lower vascular resistance.

$-{ }_{-}=$Responders; - - - = non-responders

not given to patients who regularly engage in endurance exercise.

The hemodynamic picture at the opposite pole, when the cardiac output is high, can be equally disturbing. In spite of the lower afterload, the large increase in cardiac output increases the overall cardiac work and patients with decreased coronary reserve may develop a variety of ischemic syndromes.

\section{What is being vasodilated?}

Vasoconstrictive or vasodilating stimuli elicit very diverse responses along different segments of the vascular tree. Veins are different from arteries in regard to both the vascular geometry and the contractile properties of the smooth muscles. The wall-to-lumen ratio in the resistance

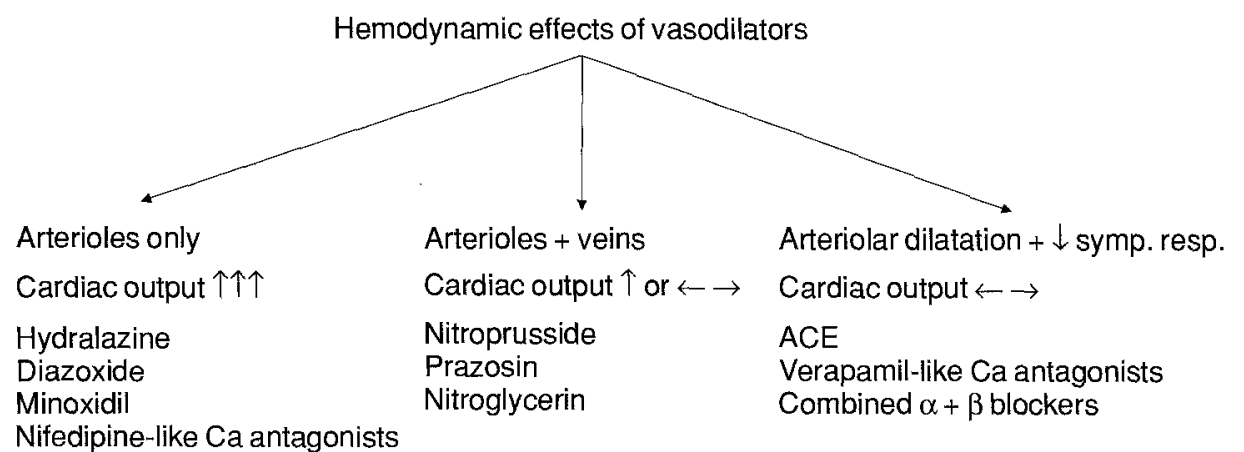

Nifedipine-like $\mathrm{Ca}$ antagonists

Fig.3. See text 
vessels is much larger, and vasodilation causes a large change in the vascular resistance. A similar decrease in venous smooth-muscle contraction does not affect the resistance; the response is a large increase in vascular capacity. The balance between hemodynamic changes in the resistance and capacitance vessels determines the level of cardiac output.

A drug causing only arteriolar dilatation, with no increase in venous capacity, reduces the blood pressure but simultaneously increases the cardiac output. As mentioned above, an excessive cardiac output may have negative clinical effects. A predominant venodilator decreases the preload and thereby reduces the cardiac output. This is particularly bothersome in the upright posture, since excessive gravitational pooling of blood frequently causes orthostatic hypotension. A schematic overview of the effect of various classes of vasodilators on cardiac output is given in Fig. 3.

Veins and arterioles are morphologically very different, and it is easy to visualize why they would be functionally different. The morphology of arterioles across the whole body is rather uniform. Nevertheless, arterioles show a great range of functional differences and their response to vasodilators varies from organ to organ. These differences in regional vasodilation must be taken into consideration when one evaluates the clinical profile of a vasodilator. Unpleasant flushing and peripheral edema with some calcium antagonists, as well as headaches with hydralazine, reflect the excessive regional vasodilation caused by these compounds. The substantial fluid retention observed with minoxidil is in large part caused by unfavorable redistribution of the renal blood flow.

\section{Which compensatory responses are elicited?}

Vasodilation triggers a number of counterregulatory responses; some of them directly oppose the vasodilation, whereas others strive to maintain an unchanged blood pressure. The pressure-preserving mechanisms of particular interest are sympathetic cardiostimulation, fluid retention, and activation of the renin-angiotensin system.

Acute vasodilation inevitably elicits reflex increases in the heart rate and stroke volume. In addition to the aforementioned balance between dilatation of the veins and arterioles, this reflex cardiostimulation largely determines the degree of elevation of the cardiac output. This increased sympathetic drive may have a specific negative effect on the heart. The issue evolves around the socalled trophic effect of the sympathetics. Whereas the increase in blood pressure mechanically elicits cardiac and vascular hypertrophy, the degree of hypertrophy depends on various other "growth" factors. Denervated blood vessels fail to develop vascular hypertrophy $[5,6]$. In spontaneously hypertensive rats, reflex-inducing vasodilators (hydralazine and minoxidil) decrease the blood pressure but fail to reduce the left ventricular hypertrophy [7]. Rats treated with a sympatholytic agent $(\alpha-$ methyldopa) showed significant reduction of the left ventricular wall mass in spite of poorer blood pressure control. Noradrenaline facilitates the growth of cardiac myocytes in isolated culture [8]. This effect appears to be regulated through alpha-adrenergic adrenoreceptors. Left ventricular hypertrophy is a strong risk factor for future blood-pressure-related mortality and morbidity. Since reflex-inducing vasodilators favor ventricular hypertrophy, they are not the preferred treatment of choice for hypertension.

Fluid retention is another potent counterregulatory mechanism to offset the hypotensive effect of vasodilators. This is due in part to the redistribution of the intrarenal blood flow, but activation of the renin-angiotensin system also plays a major role. If the fluid retention is not prevented, a vasodilator will lose its initial efficacy. Drugs that directly antagonize the reninangiotensin system (converting enzyme inhibitors) or prevent the reflex increase in renin (combined vasodilators-beta-blockers) greatly obviate the problem. Calcium antagonists also cause little fluid retention as, in addition to causing vasodilation, they also directly increase diuresis.

\section{What is an ideal vasodilator?}

Vasodilators are not a homogeneous group of compounds with uniformly advantageous features. To be efficacious and useful, a vasodilator must fulfill certain criteria:

1. Vasodilation should occur at a normal level of cardiac output; levels that are either too low or too high are undesirable.

2. The vasodilator should not cause excessive blood flow in the skin or in other nonessential organs.

3. Renal blood flow should not be reduced.

4. There should be little or no fluid retention.

5. Vasodilators should either not elicit or be capable of abolishing sympathetic counterregulatory responses.

6 . The vasodilator should notcause excessive venodilation.

Three major groups of contemporary vasodilators meet most of these criteria: converting enzyme inhibitors, some calcium antagonists, and combined alpha/beta-adrenergic blocking agents. Converting enzyme inhibitors are not direct vasodilators; rather, they remove the angiotensin-mediated vasoconstriction. Nevertheless, the end result is a decrease in vascular resistance. This decrease is not associated with tachycardia or with an increase in plasma norepinephrine [9]. Converting enzyme inhibitors antagonize the effect of angiotensin on aldosterone and thereby prevent sodium and fluid retention.

Dihydropiridine-type calcium antagonists are potent peripheral vasodilators and elicit very strong sympathetic responses. The clinical side effects of headache, tachycardia, and flushing can limit their usefulness. Diltiazem and verapamil-like drugs, although generally less efficacious as dilators, can prevent tachycardia; they are also mild diuretics. When edema occurs, this is due to fluid redistribution from the intravascular to the interstitial space and not to generalized fluid retention. Absence of both fluid retention and tachycardia are desirable features.

Combined alpha- and beta-adrenergic blocking agents are a welcome addition to our antihypertensive armamentarium. Their alpha-adrenergic blocking action is respon- 
sible for vasodilation, whereas the beta-blocking component adds other desirable features. Blockade of cardiac and renal beta-adrenergic receptors prevents tachycardia and the renin-angiotensin-induced sodium retention. The clinical efficacy of these compounds depends on whether the duration and potency of the alpha- and beta-adrenergic component at a given dose are well balanced. Other ancillary properties such as the magnitude of the venous effect and the presence of intrinsic sympathomimetic activity are also important. These differences require that each new compound in this class be examined in its own right. The drug that is the subject of this symposium, carvedilol, has a number of attractive properties. The vasodilation in the skin and muscles is not excessive, the decrease in peripheral resistance occurs at normal levels of cardiac output, and there is no evidence of excessive venodilation or fluid retention.

The efforts of the pharmaceutical industry to bring new compounds to the market may confuse the practicing physician with new names and doses to learn. However, many of these compounds offer a true advantage. Generally speaking, the more uniform a class of drugs (diuretics, converting enzyme inhibitors), the less likely it is that a new compound will be truly different. However, within the class of combined beta- and alpha-blocking agents, much depends on the balance between various characteristics of these multiple-action compounds. The impressive dossier of clinical trials and some of the interesting features of carvedilol make it a compound well worth consideration in everyday practice.

\section{References}

1. Julius $S(1988)$ The blood pressure seeking properties of the central nervous system. J Hypertension 6:177-185

2. Julius S, Egan B (1986) Hemodynamics of hypertension. In: Zanchetti A, Tarazi RC (eds) Handbook of hypertension, vol 7. Pathophysiology of hypertension - cardiovascular aspects. Elsevier, Amsterdam, pp 153-178

3. Strandgaards OJ, Skinhoj E, Lassen NA (1973) Autoregulation of brain circulation in severe arterial hypertension. Br Med J 1: $507-510$

4. Hansson L, Zweifler AJ, Julius S, Hunyor SN (1974) Hemodynamic effects of acute and prolonged $\beta$-adrenergic blockade in essential hypertension. Acta Med Scand 196: 27-34

5. Bevan RD (1975) Effect of sympathetic denervation on smooth muscle cell proliferation in the growing rabbit ear artery. Circ Res 37: $14-19$

6. Hart MN, Heistad DD, Brody MJ (1980) Effect of chronic hypertension and sympathetic denervation on the wall/lumen ratio of cerebral vessels. Hypertension 2: 419-428

7. Sen S, Tarazi RC, Bumpus FM (1977) Cardiac hypertrophy and antihypertensive therapy. Cardiovasc Res 11: 427-433

8. Simpson P (1983) Norepinephrine-stimulated hypertrophy of cultured rat myocardial cells in an alpha $\mathrm{a}_{1}$-adrenergic response. J Clin Invest 72: 732-738

9. Ibsen H, Egan B, Julius S (1983) Baroreflex sensitivity during converting enzyme inhibition with Enalapril (MK-421) in normal man. J Hypertens 1 [Suppl 2]: 222-224

S. Julius, M.D., Sc. D.

Division of Hypertension

Department of Internal Medicine

University of Michigan Medical Center

3918 Taubman Center

Ann Arbor, Michigan 48109-0356, USA 